\title{
CUESTIÓN DE AMENAZA: LA INFLUENCIA DE LOS FACTORES DOMÉSTICOS EN EL COMPORTAMIENTO DE VIETNAM Y MALASIA ANTE EL ASCENSO DEL PODER MILITAR CHINO
}

\author{
A QUESTION OF THREAT: THE INFLUENCE OF \\ DOMESTIC FACTORS ON THE BEHAVIOR OF VIETNAM \\ AND MALAYSIA IN THE FACE OF THE RISE OF \\ CHINESE MILITARY POWER
}

\section{Lautaro Nahuel Rubbi ${ }^{1}$ \\ ORCID: 0000-0003-0025-9744}

\section{Evelin Martínez Schandeler ${ }^{2}$ \\ ORCID: 0000-0003-4249-0995}

\section{RESUMEN}

En las últimas décadas China aumentó enormemente su poder, tanto en términos económicos como militares. Sin embargo, la respuesta de los actores de la región no fue homogénea; mientras que algunos recurrieron al balance de poder y el aumento de sus capacidades, otros evitaron este tipo de comportamiento. Entre 2013 y 2018, Vietnam elevó su gasto militar de forma continua, mientras que Malasia llegó incluso a disminuir el suyo, planteando un interrogante importante para el realismo estructural. A través de un estudio comparado entre ambos casos se concluye que el realismo neoclásico, que incorpora las creencias y las percepciones de los respectivos gobiernos y sociedades al análisis, ofrece una mejor explicación de los acontecimientos. En estos casos, las variables domésticas de cada Estado moldearon el accionar de sus gobernantes y jugaron un rol central en la percepción de China como una amenaza y la consecuente estrategia de respuesta ante su ascenso.

Palabras clave: China; Vietnam; Malasia; Balance de poder; Realismo neoclásico; Percepción del gobierno.

\section{ABSTRACT}

In recent decades, China increased its power greatly, both in economic and military terms. However, the response from the actors in the region was not homogeneous; while some of them appeal to balance of power and increase their capabilities, others avoid this behavior. Between 2013 and 2018, Vietnam continuously increased

1 Universidad Argentina de la Empresa, Instituto de Ciencias Sociales y Disciplinas Proyectuales, Buenos Aires, Argentina. Docente investigador y Magister en Estudios Internacionales. Correo: 1rubbi@, uade.edu.ar

2 Universidad Argentina de la Empresa, Buenos Aires, Argentina. Licenciada en Gobierno y Relaciones Internacionales. Correo: evmartinez@uade.edu.ar 
its military expenditure, while Malaysia even decreased its own, representing an important question for Structural Realism. Through a comparative study between the two cases, it is concluded that Neoclassical Realism offers a better explanation of the events, incorporating government and societies' beliefs and perceptions into the analysis. In these cases, each State domestic variables shaped the actions of their governing authorities and played a central role in the perception of China as a threat and the consistent response strategy to its rise.

Keywords: China; Vietnam; Malaysia; Balance of power; Neoclassical Realism; Government's perception.

\section{Introducción}

Ante el deterioro relativo de la hegemonía norteamericana en las últimas décadas, China se ha posicionado como el principal actor en ascenso en el Asia Pacífico. La distribución de las capacidades materiales está cambiando, dando lugar a una competencia en términos militares que desestabiliza la región (Liff y Ikenberry, 2014). Potenciados por su activo rol de liderazgo durante la crisis internacional del 2008, los gobernantes chinos perciben, desde entonces, que su oportunidad finalmente ha llegado, y comienzan a actuar de manera más asertiva (expresan las opiniones, las actitudes y los deseos, y reclaman los propios derechos) y, en oposición al orden establecido, promueven sus propias normas y políticas alternativas en muchos aspectos (Swaine, 2010).

Además, el desarrollo militar de China se halla ligado, de forma directa, a su crecimiento económico. Según el Instituto Internacional de Estocolmo para la Investigación de la Paz (SIPRI, 2019a), entre el año 2013 y el 2018 el gasto mili$\operatorname{tar}^{3}$ del país se incrementó casi un $36 \%$, y se mantuvo estable con relación a su PBI, de constante aumento, lo que lo convirtió en la principal potencia militar de la región y la segunda a nivel mundial (Beckley, 2017).

Considerando este escenario, es esperable observar, desde la perspectiva del realismo estructural, tanto en su vertiente defensiva como ofensiva, un aumento del gasto militar en todos los países de la región, obligados a procurar el mantenimiento del balance de poder ${ }^{4}$ en un mundo anárquico (Mearsheimer, 2001; Waltz, 1979). No obstante, esto no ha sucedido; o al menos no en todos

3 Todas las cifras del gasto militar son expresadas en dólares norteamericanos constantes al 2017.

4 El concepto de balance de poder está ligado al concepto de anarquía. La ausencia de una autoridad central en el sistema internacional obliga a los Estados a actuar de manera agresiva entre sí, en busca de su propia seguridad (Mearsheimer, 2001). Ante una amenaza, para maximizar la seguridad o su participación en el poder mundial, los Estados tienden a incrementar su poder a expensas de posibles rivales (mediante recursos económicos, diplomáticos y militares), con el fin de alterar el balance de capacidades. Dado que el concepto de equilibrio de poder es muy amplio y abarca múltiples dimensiones, el presente trabajo se centra en el balance de poder duro (militar) interno y externo. El balance de interno supone que los Estados pueden equilibrarse contra un potencial agresor movilizando los recursos propios, mientras que el balance externo implica la formación de alianzas con otros Estados. 
los casos. Contrario a lo propuesto por esta teoría, Malasia, Myanmar y Brunéi disminuyeron su gasto militar (SIPRI, 2019a), planteando importantes interrogantes al paradigma realista. Cabe preguntarse, esencialmente, qué explica estas diferencias de comportamiento.

Figura 1

Variación en el gasto militar de Vietnam y Malasia entre 2013 y 2018 en millones de USD

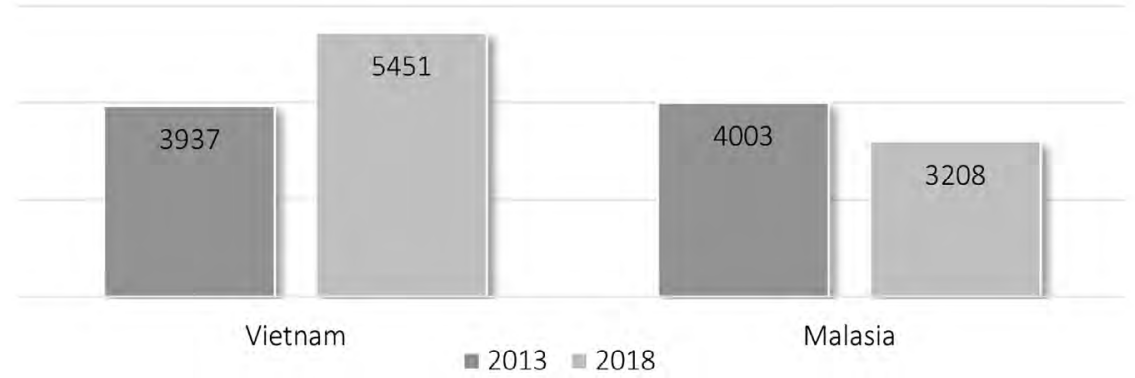

Nota: SIPRI (2019) Military Expenditure Database.

El presente artículo aborda los casos de Vietnam y Malasia como dos casos extremos de la región durante el período comprendido entre 2013 y 2018, a fin de arrojar luz sobre las razones de este comportamiento. En consonancia con datos de SIPRI (2019a), en este período Vietnam incrementó su gasto militar de USD 3937 millones en 2013, a USD 5451 millones en 2018, lo cual representa un aumento del $38 \%$ y aumenta el gasto en términos de porcentaje de PBI desde 2014 hasta 2016. A la inversa, Malasia presentó un gasto militar en 2013 de USD 4 003 millones (representa el 1,5\% del PBI) y USD 3208 millones en 2018 (casi el $1 \%$ de su PBI), por lo que se produjo una disminución aproximada del $20 \%{ }^{5}$.

Se plantea como hipótesis que las variables domésticas que incorpora el realismo neoclásico, esencialmente la percepción de amenaza por parte de los gobiernos, actuaron en ambos casos como mediadores entre la presión estructural y las definiciones en términos de política exterior. A mayor amenaza percibida es

5 Durante 2015 se registró en Malasia un aumento del gasto militar, que aún representa, como en 2013 y 2014 , el 1,5 \% del PBI. El incremento se explica por la desaceleración de la economía china y la debilidad en los precios de los commodities que derivó en la devaluación del ringgit. Pero, a partir del 2016, el gasto militar disminuyó incluso en términos de porcentaje del PBI, y llegó al $1 \%$ en 2018 (Cf. Parameswaran, 2016). 
más probable observar comportamientos en torno al balance de poder interno y externo para responder a esta, como sintetiza la Tabla 1.

Tabla 1

Comparación de la percepción del gobierno y el balance de poder interno en Vietnam y Malasia ante el aumento de poder de China (2013-2018)

\begin{tabular}{|c|c|c|}
\hline & VIETNAM & MALASIA \\
\hline $\begin{array}{l}\text { PRESIÓN } \\
\text { ESTRUCTURAL }\end{array}$ & \multicolumn{2}{|c|}{$\begin{array}{l}\text { Aumento del poder chino en términos económicos y } \\
\text { militares. Relación asimétrica y dependiente en términos } \\
\text { económicos }\end{array}$} \\
\hline $\begin{array}{l}\text { PERCEPCIÓN DEL } \\
\text { GOBIERNO }\end{array}$ & $\begin{array}{l}\text { Relativamente } \\
\text { desfavorable - respuesta } \\
\text { al nacionalismo de la } \\
\text { sociedad civil }\end{array}$ & $\begin{array}{l}\text { Favorable - respuesta a } \\
\text { las minorías étnicas chinas } \\
\text { y a la priorización de la } \\
\text { relación económica }\end{array}$ \\
\hline REACCIÓN & $\begin{array}{l}\text { Aumento de capacidades } \\
\text { militares }\end{array}$ & $\begin{array}{l}\text { Disminución del gasto en } \\
\text { capacidades militares - } \\
\text { acercamiento diplomático }\end{array}$ \\
\hline
\end{tabular}

Nota: Elaboración propia.

Para corroborar esta hipótesis, se plantea la utilización del método de comparación controlada entre ambos casos mencionados. Luego de presentar la metodología y profundizar sobre las premisas del realismo neoclásico, se describe el ascenso de China, considerando sus capacidades económicas y militares. Seguidamente se aborda la comparación del comportamiento de Malasia y Vietnam, contextualizando sus desarrollos militares en el marco de sus creencias y percepciones sobre el gobierno de Xi Jinping como factor explicativo de sus acciones. Se finaliza planteando algunas limitaciones del análisis y futuras líneas de investigación ${ }^{6}$.

\section{Metodología}

Este artículo recurre a un diseño de alcance explicativo basado en el método comparado de "sistemas más similares", analiza casos que son lo más similares posible en todos los aspectos, exceptuando la variable de interés, donde se espera que exista una variación como explicación de los resultados divergentes (Gerring, 2001).

6 Quienes escriben este artículo agradecen los comentarios rigurosos, detallados y pertinentes de los pares revisores y del grupo editorial de la Revista Relaciones Internacionales de la Universidad Nacional, Costa Rica. Sus sugerencias han ayudado a optimizar la calidad del artículo. 
En lo que respecta a las variables de análisis, se considera el efecto de la presión estructural del Sistema Internacional (Variable Independiente) sobre la política exterior estatal (variable dependiente). Esta última se mide fundamentalmente a partir de la respuesta en términos de desarrollo militar interno y búsqueda de alianzas externas (balance de poder), así como el comportamiento en términos diplomáticos de ambos actores. Como propone la teoría del realismo neoclásico, se incorporan también aspectos domésticos como variable interviniente y mediadora de la relación entre ambas. Se avanza fundamentalmente sobre la dimensión de percepción de amenaza a nivel de los cuerpos gubernamentales decisores y de la población en general, así como la relación entre ambos actores a nivel interno, tomando indicadores de distintas encuestas de opinión pública y hechos de movilización social, así como declaraciones públicas de de personal en puestos altos.

Sobre la selección de casos de comparación, se tomaron dos casos extremos y opuestos en términos de la variable dependiente, fueron los casos de mayor aumento y descenso en el gasto militar anual durante el período analizado en la región (además de China, que se plantea como condicionante estructural).

Además, los casos de Malasia y Vietnam cumplen con las condiciones necesarias para la comparación controlada de sistemas más similares, en tanto se equiparan en diversas variables que podrían considerarse explicativas del fenómeno en cuestión. Por un lado, ambos son miembros de la ASEAN y forman parte de la región del Sudeste Asiático, limitan geográficamente con China y están en disputa territorial en el Mar del Sur de China (Schweller, 2018).

Se asemejan también en contar con sistemas políticos con partidos dominantes: en Vietnam, el Partido Comunista está en el poder desde la fundación de la república en 1976; mientras que en Malasia gobernó la misma coalición política, el Frente Nacional, desde la independencia en 1957 hasta mayo de 2018. En cuanto al sistema económico, los dos países sostienen una economía de mercado $^{7}$ (Phi, 2012). En último lugar, ambos países mantienen relaciones con los Estados Unidos en términos de seguridad y defensa. Ninguna de estas características bastaría para explicar sus diferencias de comportamiento frente al ascenso de China.

Ante estas semejanzas en aspectos geográficos, políticos y económicos, se propone que son las creencias y las percepciones de amenaza como variable

7 A inicios de la década pasada, la facción más progresista del Partido Comunista de Vietnam abogó por medidas para que el país profundizara su integración en la economía global. Los acuerdos de libre comercio, tanto bilaterales como regionales, ejercieron una presión significativa sobre Vietnam para que adoptara una economía de libre mercado (Pham, 2016). Finalmente, en 2013 se otorgó carácter constitucional a la economía de libre mercado. 
interviniente las que explican las diferencias de comportamiento entre los casos seleccionados, frente al fenómeno único del ascenso de China en términos económicos y militares.

El recorte temporal del análisis abarca el período comprendido entre marzo de 2013, con la asunción de Xi Jinping en China (que iguala las presiones estructurales en ambos casos), hasta mayo de 2018, cuando el Frente Nacional malayo perdió las elecciones ante el triunfo de la coalición del Pacto de Esperanza. De esta forma, se abarca la presidencia de Xi Jinping con el Partido Comunista de China, dos presidencias del Partido Comunista de Vietnam (Trương Tấn Sang y Trần Đại Quang) y el gobierno de Najib Razak del Frente Nacional en Malasia.

A continuación, se desarrolla, con mayor profundidad, el marco teórico que da lugar a las variables de estudio para luego avanzar sobre la profundización de cada una de estas.

\section{Realismo neoclásico: Estructura y percepción}

A fin de explicar cómo y por qué los actores de la región asiática han respondido de diversas maneras frente al mismo estímulo estructural, este artículo aborda un análisis desde el realismo neoclásico, evolución teórica del realismo estructural, que integra el análisis de las variables domésticas, junto con los principales postulados del paradigma realista (sistema anárquico y énfasis en actores estatales, entre otros), a fin de arrojar luz sobre las decisiones de política exterior ${ }^{8}$.

El realismo neoclásico se diferencia del realismo estructural al tomar en consideración diversas variables internas, supera la concepción del Estado como una "caja negra" (Lobell et al., 2009, p. 18), y reconoce que la política exterior de un Estado depende tanto de su poder material relativo como de otras características internas (Lobell et al., 2009). Si bien le otorga importancia a la estructura del sistema internacional y a la distribución de poder como variables independientes para explicar el comportamiento estatal en términos de política exterior, considera el proceso de política doméstica como una variable interviniente y moderadora de la relación entre ambas. Según esta corriente, la percepción de los líderes y grupos de poder puede modificar las respuestas por parte de los entes gobernantes ante los incentivos del sistema (Ripsman et al. 2016).

8 A partir del del análisis de los aportes de Thomas Christensen, Randall Schweller, William Wolforth y Fareed Zakaria, Gideon Rose identifica la existencia de una escuela de pensamiento unificada que incorpora la dimensión doméstica, junto con las consideraciones sobre la estructura, se aparta, así, de la escuela de pensamiento realista estructural, que tendía a desconocer la primera variable (Rose, 1998) 
Esta escuela teórica también ha profundizado sobre la cuestión de la amenaza percibida como factor fundamental para explicar la acción de balance de poder. Para todo el paradigma realista, que tiende a interpretar la competencia por el poder en pos de la seguridad en un contexto anárquico ${ }^{9}$, el concepto de balance de poder es esencial.

Schweller define el balance de poder como la "creación o agregación del poder militar a través de la movilización interna o la formación de alianzas para prevenir o disuadir la ocupación y dominación del Estado por parte de una potencia o coalición externa..." (2016, p. 6). Según la teoría, en un contexto de autoayuda, a medida que un Estado aumenta su poder, otros actores del sistema internacional se sienten amenazados y tenderán a conformar una coalición que actúe como contrapeso o desarrolle políticas de movilización de recursos internos para elevar sus capacidades ofensivas o defensivas, a fin de disuadir acciones agresivas por parte de terceros. Para sobrevivir en un contexto internacional anárquico, los Estados necesariamente deben actuar de acuerdo con esta lógica.

Sin embargo, como advierte Walt (1985), el poder por sí solo no es el único factor que implica una amenaza. Los Estados no forman alianzas únicamente como una respuesta al poder acumulado, sino principalmente contra un poder que es percibido como una amenaza. Una serie de factores afectan el cálculo de esta decisión: el poder agregado, la proximidad, las capacidades y las intenciones ofensivas. En primer lugar, el poder agregado comprende el total de recursos de un país: población, tecnología y capacidad industrial, poder militar, entre otros. La proximidad geográfica también es un factor importante, dado que los Estados de la región suponen una amenaza mayor que aquellos que están lejos. Un tercer factor es el poder ofensivo: es más probable que Estados que cuenten con capacidades de este tipo generen temor sobre otros actores que Estados con capacidades fundamentalmente defensivas. En último lugar, las intenciones ofensivas resultan cruciales ya que, cuanto más agresivo o expansionista sea un Estado, más probable es que provoque una coalición o alianza opuesta (Walt, 1985). Todas estas cuestiones son advertidas, interpretadas y ponderadas por los actores domésticos, lo que torna esencial su análisis.

9 La anarquía es la ausencia de un una autoridad central o gobierno a nivel internacional. A diferencia del interior de los Estados -que mantienen un orden jerárquico-, el estado natural del sistema internacional es la guerra o la posibilidad constante de ella, que puede estallar en cualquier momento (Waltz, 1979). Por lo tanto, la anarquía es entendida como un "extremo de un continuo, cuyo otro extremo está marcado por la presencia de un gobierno legítimo y competente ... . Se considera que la anarquía significa no solo la ausencia de gobierno, sino también la presencia de desorden y caos" (Waltz, 1979, p. 47 , traducción propia). 
En línea con esto, la incorporación de la variable doméstica permite superar el carácter prácticamente automático de respuesta mediante balance (interno o externo) que presuponía el realismo estructural ante la existencia de amenazas y la posibilidad de conflictos. En este sentido, Brooks (1997) propone una distinción entre los conceptos de posibilidad y probabilidad. Según el autor, mientras que el realismo estructural sostiene que el accionar de los Estados es moldeado ante la posibilidad de conflicto -lo que lleva a asumir que la inseguridad es persistente-, el realismo neoclásico plantea que los Estados (o más bien sus elites decisoras) toman decisiones basándose en la probabilidad percibida de una amenaza, lo que no presupone la inseguridad como una constante. De esta forma, desde el realismo neoclásico, las respuestas asumidas por los Estados dependerán de la percepción de riesgo en términos de la probabilidad de conflicto con aquellos actores que se perciben como una amenaza y no únicamente por la posibilidad (por mínima que sea) de conflicto, lo que habilita respuestas diferenciadas y no uniformes.

Este artículo propone que el realismo neoclásico provee las herramientas necesarias para explicar cómo y por qué Vietnam y Malasia han respondido, durante el período seleccionado, de manera distinta frente al mismo estímulo estructural: el aumento de poder por parte de China. A continuación, se aborda un análisis desde las variables y los conceptos planteados desde esta corriente, profundizando en el caso de la variable doméstica sobre la dimensión de la percepción gubernamental y la dimensión de la opinión pública.

\section{El aumento de poder de China: Del poder eco- nómico al poder militar}

Los recursos económicos de un país se constituyen como la base necesaria para soportar un gran desarrollo militar. Existe una conexión entre el ascenso económico de una gran potencia y su crecimiento como poder militar relevante $\mathrm{y}$, a la inversa, entre la caída a nivel económico de una gran potencia y su declive como poder militar (Mearsheimer, 2001).

Frente al actual reordenamiento económico del sistema internacional, mientras que la hegemonía norteamericana descansa actualmente sobre su supremacía militar, que le permite tener el comando de los bienes comunes: mar, espacio y aire; China ha desarrollado una estrategia de modernización militar centrada en las capacidades de "anti-acceso" y "negación de área"10, que complica la posición

10 Estas estrategias implican la limitación de la capacidad de movimiento por parte de una potencia militar competidora, en espacios cercanos al área de interés. Negar el acceso a estas áreas no supone 
de los Estados Unidos en Asia. De este modo, Beijing se posiciona como una amenaza asimétrica y podría desplegar capacidades competentes para destruir la infraestructura norteamericana en la región (Rubbi, 2018).

El inicio del ascenso de China como gran potencia se fundamentó sobre el despliegue de su poder económico. En 1979 comenzó la implementación de reformas de libre mercado y la apertura al comercio exterior. Desde entonces, el país se posicionó entre las economías de más rápido crecimiento a nivel mundial (Morrison, 2014). Ante la crisis asiática en 1997, China vio la oportunidad de expandir sus compromisos con Asia y llevó a cabo una política de acercamiento mediante el fomento de vínculos comerciales y de la integración regional (Ku, 2017).

Esta política se intensificó con la asunción de Xi Jinping en 2013, cuyo gobierno demostró, a diferencia de sus antecesores, una "mayor asertividad, confianza y disponibilidad de tomar liderazgo ante el sistema internacional" (Vélez Serrano, 2018, p. 3). Mediante la doctrina del "ascenso pacífico ${ }^{11}$ ", el gobierno pretende convencer al mundo de que su progreso no representa una amenaza para el orden internacional y, mientras tanto, se beneficia de un entorno favorable para su desarrollo económico (Chen y Feffer, 2009). En este marco, el mandatario chino lanzó dos iniciativas destinadas a fomentar el desarrollo del país: el plan One Belt, One Road (OBOR) y el plan Made in China 2025 $5^{12}$. Ambos resultan un instrumento diplomático e ilustran cómo China acepta su rol en el sistema, apoyando el comercio internacional y, al mismo tiempo, manifestando su intención de adoptar un papel activo (Rocha Pino, 2020).

De acuerdo con la lógica realista, el aumento del poder económico conlleva un aumento de poder militar. En el caso de China, el objetivo de su modernización militar iniciada a mediados de 1990 consistió en transformar su enorme ejército en uno capaz de ganar conflictos de corta duración y alta intensidad en su propia periferia, contra adversarios con fuerzas altamente modernizadas. Los motivos que influyen en la modernización de las fuerzas militares de China son

una proyección de poder por sí misma, sino que pretende la limitación o incluso la anulación de la capacidad de acceso del otro (Battaleme, 2015)

11 El término hace referencia a un proceso en que el Estado en crecimiento se adapta a las reglas y estructuras del sistema internacional, al tiempo que las otras potencias se acomodan en torno a los nuevos cambios que produce. Esto implica que el país en ascenso logra obtener ganancias, tanto materiales como de estatus, sin generar grandes hostilidades con las otras potencias (Buzan y Cox, 2013).

12 A fines de 2013, Xi Jinping anunció la construcción del cinturón económico "One Belt, One Road", proyecto de infraestructura que pretende conectar las regiones fronterizas de China con los países de la región, con la finalidad de fortalecer la integración económica y favorecer el desarrollo (Cai, 2017). Dos años después, el gobierno lanzó el plan "Made in China 2025", un programa de autosuficiencia que establece diez industrias clave para el país, en las que se espera que las empresas nacionales controlen hasta el $80 \%$ del mercado interno y planteen su proyección internacional (Economy, 2018). 
la necesidad de garantizar el acceso a recursos naturales, las disputas territoriales con países vecinos y, a nivel interno, la necesidad de mantener la cohesión y prosperidad económica (Chen y Feffer, 2009).

China cuenta con disputas marítimas y territoriales: reclama su soberanía sobre más del $80 \%$ del Mar del Sur de China, abarcando aguas que también son reclamadas por Vietnam, Malasia, Filipinas, Brunéi e Indonesia; está en disputa con Japón en el Mar Oriental de China, y con Taiwán por tensiones en la frontera y, de forma más general, por el reconocimiento del principio de "una sola China". Las disputas territoriales juegan un papel clave en el pensamiento geopolítico, que lleva al país a promover el aumento del gasto militar, con el fin de impulsar y defender estos reclamos (Chen y Feffer, 2009).

Por otro lado, resulta fundamental que un Estado cuente con una población de considerable magnitud, ya que posibilita y promueve el dinamismo económico $\mathrm{y}$, en consecuencia, la riqueza. Aunque no supone un factor determinante, una gran población constituye un factor que condiciona la construcción de fuerzas militares (Mearsheimer, 2001). China cuenta con esta ventaja dado que tiene 1 393 millones de habitantes, es el Estado más poblado del orbe (Banco Mundial, 2019b), lo que contribuye a la confirmación de uno de los ejércitos más grandes del mundo. Si bien Beijing aún tiene escaso margen de maniobra para intervenir en conflictos por fuera de su región, está más que capacitada para la defensa de sus intereses en el ámbito cercano (Rubbi, 2018).

Figura 2

Evolución del PBI y gasto militar de China desde 2013 a 2018

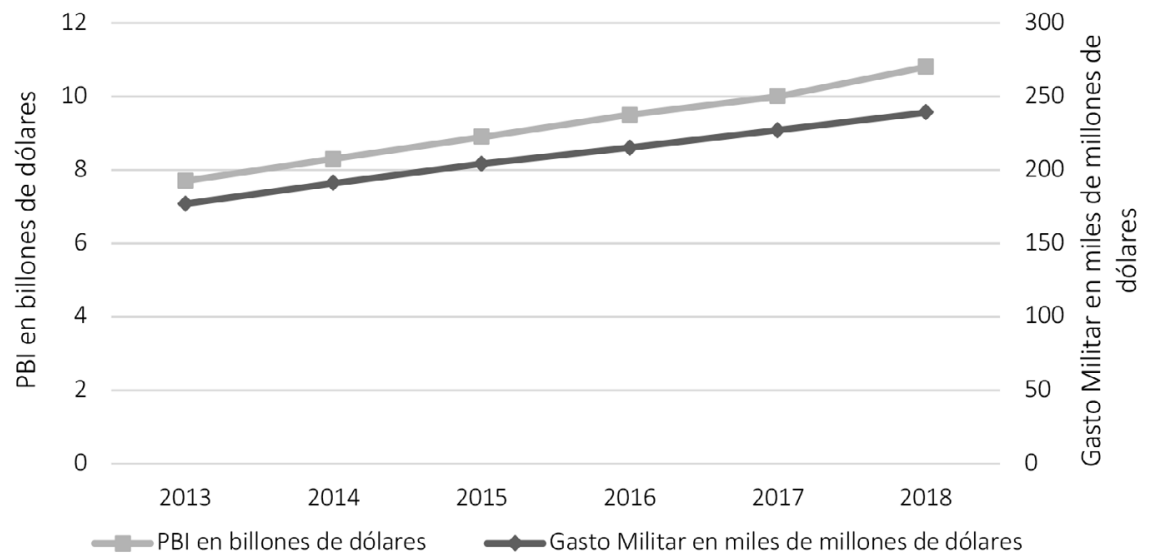

Nota: Elaboración propia a partir de datos de Banco Mundial (2019) y SIPRI (2019). 
Como se nota en la Figura 2, el gasto militar de China está ligado al crecimiento económico: en 2013 China presentó un $\mathrm{PBI}^{13}$ de 7,7 billones de dólares y alcanzó los 10,8 billones para 2018 (Banco Mundial, 2019a), mientras que su gasto militar aumentó durante este período de 179 mil millones de dólares en 2013 a 249 mil millones en 2018. De este modo, el gasto militar se mantuvo en forma estable con respecto al desarrollo económico, siempre cerca del $2 \%$ del PBI anual $^{14}$ (SIPRI, 2019a). Sin embargo, en relación con el gasto militar global, el porcentaje del gasto militar chino creció de forma significativa, en 2008 fue del 5,8 \%, y alcanzó ya el $13 \%$ en 2017 (SIPRI, 2018a).

La modernización de las fuerzas militares implicó que el Ejército Popular de Liberación se convirtiera en la fuerza militar más potente de Asia, capaz de obstaculizar maniobras militares de Estados Unidos ante las disputas en la región (Storey, 2018).

Ante este escenario, que generó presiones estructurales y se podría haber configurado como una amenaza a la seguridad estatal de sus vecinos, los actores de la región han respondido de forma diversa, en tanto sus comportamientos se vieron condicionados por lineamientos internos. A continuación, se desarrolla la comparación entre los casos de Vietnam y Malasia, a fin de dar cuenta de esta perspectiva.

\section{El caso de Vietnam: Balance interno ante el aumento de poder de China}

La relación entre China y Vietnam es considerada una relación definitoria para la política regional del Sudeste Asiático. Con el fin de la Guerra de Vietnam, las relaciones entre ambos Estados se vieron deterioradas y comenzaron las disputas territoriales por el Golfo de Tonkin y por el Mar del Sur de China y llevaron al ataque chino a Vietnam en 1979. En 1991 se normalizaron las relaciones y, a partir de entonces, se caracterizaron por dos tendencias contradictorias: por un lado, se produjo un aumento de cooperación en el ámbito político, cultural, económico y militar; por el otro, se mantuvo la disputa territorial (Amer, 2004).

Las relaciones entre ambos países han sido históricamente asimétricas, independientemente de si han sido hostiles, amistosas o neutrales (Womack, 2006);

13 Todas las cifras referentes al PBI son expresadas en dólares norteamericanos constantes de 2010.

14 Si bien China anuncia públicamente su presupuesto militar, analistas externos consideran que tiende a subestimar sus gastos militares, por lo que los datos provistos por SIPRI no se condicen con los oficiales (Chen y Feffer, 2009) 
Vietnam ha debido mantenerse al tanto de la política exterior y la política doméstica de China para evaluar los costos y oportunidades de converger o disentir con el país (Nguyen, 2016). Esta asimetría es clara en materia económica. Dado que China ha sido por mucho el mayor socio comercial de Vietnam durante años, el crecimiento económico vietnamita no puede sostenerse sin la relación con Beijing (Toai y Ghimire, 2018).

Por otro lado, la liberalización económica de Vietnam y el crecimiento de su PBI desde la década de 1990, además de una exitosa integración en el mercado global y una estrategia orientada a la exportación, le otorgaron al país la oportunidad de incrementar y perfeccionar sus capacidades militares (Tran, 2018). De este modo, durante los años 2013-2018 Vietnam presentó un crecimiento económico sostenido. En 2013 su PBI fue de 136 mil millones de dólares, alcanzando en 2018 los 187 mil millones de dólares, un crecimiento del 37 \% (Banco Mundial, 2019a). A su vez, el rápido crecimiento económico de Vietnam se vio acompañado de un incremento en el gasto militar de un 38 \%. Para 2013 destinaba casi 4 mil millones de dólares al gasto militar, y alcanzó los 5 mil millones y medio en 2018 .

De acuerdo con el Instituto Internacional de Estudios Estratégicos (IISS, 2018), el aumento del gasto militar durante este período -incluso en términos del PBIfue llevado a cabo con el fin de disuadir la presión de Beijing en las disputadas Islas Spratly en el Mar del Sur de China. Para 2013, la cifra era del 2,2 \% del PBI, alcanzó en 2015 y 2016 al 2,4 \% (SIPRI, 2019a). Además, el desarrollo militar se observa también en la importación de armas, que aumentó de manera notable: entre 2007 y 2011, Vietnam se ubicaba en el vigésimo noveno puesto en el ranking de importadores de armas a nivel global, y pasó a ser el décimo en el período 2012-2018 (SIPRI, 2018b). Es importante considerar que a partir de 2014 Vietnam comienza a converger con los Estados Unidos en asuntos estratégicos, lo que culmina en 2016 con el levantamiento del embargo de armas a Vietnam (Shoji, 2018).

La primera fase de la modernización militar de Hanói comenzó a mediados de la década de 1990, con la adquisición de plataformas navales y aéreas como respuesta a la disputa en el Mar del Sur de China. La segunda fase inició en 2006 con un programa que, mediante acuerdos con diversos países, pretendió modernizar la industria de la defensa nacional y desarrollar capacidades logísticas en el Mar del Sur de China (Thayer, 2018, p. 431).

China supone, en términos de Walt (1985), una amenaza para Vietnam. En primer lugar, en cuanto poder agregado China posee amplias ventajas militares, además de ventajas en términos económicos, territoriales y demográficos. A 
esto se suma la amenaza por la proximidad geográfica, evidenciada en la disputa sobre los territorios de su Mar del Sur. Por otra parte, ciertamente China cuenta con capacidades ofensivas que pueden atentar contra la seguridad de Vietnam, que representa la mayor amenaza a sus intereses marítimos. Cabe destacar que, si bien China no es el único Estado que reclama una porción del territorio, las capacidades navales de los demás resultan limitadas, por lo que no suponen una amenaza creíble para Vietnam (Wu, 2019).

Como la capacidad naval vietnamita resulta asimétrica en comparación con China, su modernización militar se centra en el desarrollo de capacidades de antiacceso y negación de área, lo cual apunta a evitar, eventualmente, el acceso de Beijing a sus aguas territoriales (Karim y Chairil, 2016). Aunque Vietnam no puede equilibrar el poder de China, sus recientes adquisiciones de aviones de combate $\mathrm{Su}-30 \mathrm{MK} 2$ y nuevas capacidades de defensa aérea podrían generar cierto nivel de disuasión (IISS, 2018; Wu, 2019). No obstante, aun considerando la modernización y el incremento en el gasto militar, la brecha entre los recursos materiales de China y Vietnam es muy amplia, principalmente en cantidad y nivel tecnológico de sus aviones de combate, buques y submarinos.

Se puede asumir una relación causal entre el aumento de poder de China y el incremento militar de Vietnam, pero no hay consenso entre la academia al respecto: la modernización militar vietnamita puede enmarcarse dentro de una tendencia regional, o ser entendida como una combinación de su propio desarrollo económico y de la asertividad china (Strasakova, 2017; Tran, 2018). Para Karim y Chairil (2016), Vietnam es el único Estado del Sudeste Asiático que declaró que su incremento militar y acumulación de armas se debe a la acumulación naval de China.

En 2016, en el marco del $12^{\circ}$ Congreso Nacional del Partido Comunista de Vietnam, el Ministro de Defensa General Ngo Xuan Lich expresó que "Vietnam enfrentará varios desafíos y dificultades dado que se espera que la situación en el Mar del Este de Vietnam [o Mar del Sur de China] se intensifique, requiriendo más esfuerzos para construir y defender la nación" (Tuoi Tre News, 2016). En esta ocasión también afirmó la voluntad del país de "fortalecer la formación de un ejército oficial, de élite y moderno con altas sinergias y preparación para el combate, que será la fuerza fundamental en la defensa nacional" (Tuoi Tre News, 2016). De este modo, el ministro señaló que el país enfrentaría varios desafíos por una posible intensificación en el conflicto en el Mar del Este de Vietnam (Tran, 2018). Esta declaración advierte que el gobierno de Vietnam podría responder a las acciones asertivas de China con un incremento en su gasto militar. 
Tran (2018) enmarca la postura de Vietnam desde 1990 hasta 2010 en el concepto de hedging, un comportamiento que responde a la estrategia de maximizar su autonomía mediante un incremento de la defensa, y al mismo tiempo mantener buenas relaciones con los grandes poderes. Sin embargo, el creciente comportamiento agresivo de Beijing -a partir de 2010 y que se incrementa en 2014- ha presionado a Vietnam para que implemente una estrategia de equilibrio de poder más agresiva.

\section{Percepción del gobierno vietnamita y el rol de la opinión pública}

La formación de percepciones entre ambos países es definida por la memoria histórica, la política doméstica y el contexto internacional (Nguyen, 2016). El conflicto marítimo juega un papel crucial en el refuerzo de su tradicional percepción de China como una potencia agresiva, expansiva y revisionista (Strasakova, 2017). Es importante resaltar que, a pesar de que Vietnam y China son ideológicamente similares, el factor del comunismo no actuó como un componente positivo en la constitución de la percepción del gobierno y de la sociedad civil respecto de China. Al respecto, Walt (1990) afirma que la ideología no resulta determinante para las relaciones bilaterales y, de hecho, su alcance entre las superpotencias y sus aliados suele ser bastante limitado.

Con anterioridad al 2012 ya se producían en Vietnam algunas protestas espontáneas anti-China en el país, pero eran sofocadas por el gobierno (BBC, 2011). A partir de esa fecha se intensificaron las manifestaciones contra China en distintas ciudades y, cuando en mayo de 2013 China desplegó una plataforma petrolera en una zona disputada en el Mar del Sur de China, el gobierno resolvió permitir las protestas en contra de China (South China Morning Post, 2014).

Por otro lado, si bien el reclamo chino sobre el Mar del Sur de China no tiene validez a nivel internacional -ya que el Tribunal Internacional de La Haya rechazó sus reclamos en 2016-, Beijing insiste e incluso coloca al Mar Meridional como propio en todos sus mapas y pasaportes (Beckley, 2017). Ante esta situación, una parte del funcionariado vietnamita se negó a sellar los nuevos pasaportes, calificándolos como temporalmente inválidos (BBC, 2012). En este marco, las tensiones tanto en la población vietnamita como en la china aumentaron: en 2016 las pantallas de dos aeropuertos de Vietnam fueron hackeadas para mostrar mensajes con críticas a los reclamos del país en el Mar del Sur de China, como respuesta Vietnam acusó a un hacker chino como responsable (BBC, 2016). 
Datos del proyecto de Asian Barometer Survey otorgan una comparación de la opinión pública de Vietnam respecto a China en dos períodos temporales: como se aprecia en la Figura 3, entre 2010 y 2012, el 56 \% de la población de Vietnam calificó la influencia de China en su país como positiva. Mientras tanto, durante 2014 y 2016, la percepción favorable hacia China cayó al 20 \% del total (Chu y Chang, 2017). El aumento en la percepción desfavorable hacia China estuvo vinculado con la escalada de conflicto en mayo de 2014 en el Mar del Sur de China, cuando una empresa china desplegó una plataforma petrolera dentro de la zona económica exclusiva de Vietnam. En el mismo mes, un barco pesquero de Vietnam fue hundido cerca de la plataforma petrolera china en las Islas Paracel, luego de ser embestido por un barco chino; ello aumentó aún más las tensiones.

Figura 3

Percepción de la población de Vietnam hacia China entre 2010-2012 y 2014$2016(\%)$

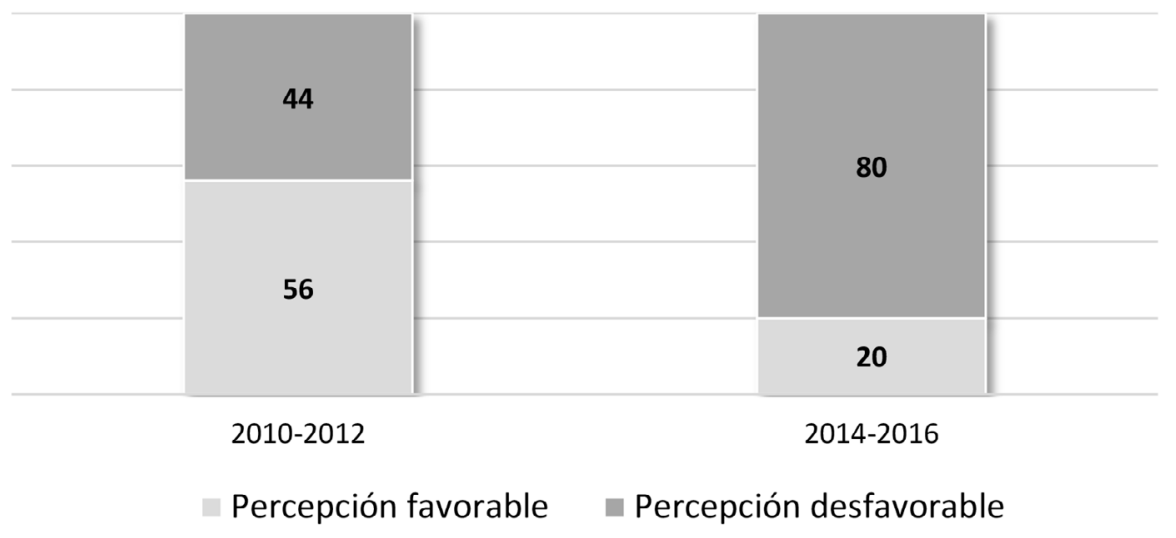

Nota: Chu y Chang (2017). Battle for Influence: Perceptions in Asia of China and the US.

La escalada de violencia intensificó los sentimientos nacionalistas y anti-China en la población (Cotillon, 2017; Tran, 2018). Días después del incidente, el gobierno de Vietnam autorizó las manifestaciones contra China (una acción inusual para un país intolerante a las protestas de la disidencia) y provocó que decenas de miles de vietnamitas salieran a las calles en las ciudades más importantes del país. Las protestas fueron espontáneas, de naturaleza ad hoc y resultaron ampliamente cubiertas por los medios de comunicación estatales (South China Morning Post, 18 de mayo de 2014). Si bien el movimiento inició de manera pacífica, posteriormente se intensificó cuando manifestantes comenzaron 
a quemar empresas chinas para mostrar su descontento. El gobierno solo tomó medidas para restablecer el orden cuando las manifestaciones se tornaron violentas (Kurlantzick, 2015; South China Morning Post, 16 de mayo de 2014).

Ante estos hechos, el creciente nacionalismo anti-China implicó un desafío al liderazgo del gobierno de Vietnam, que resolvió emitir una serie de declaraciones oficiales y conferencias de prensa para denunciar la usurpación de China. El primer ministro de Vietnam declaró que la acción extremadamente peligrosa ha amenazado directamente la paz, estabilidad, seguridad y libertad de navegación en el Mar del Este (Ministerio de Relaciones Exteriores de Vietnam, 2014a). Asimismo, tanto los acontecimientos en el Mar del Sur de China como las posteriores declaraciones de Vietnam fueron ampliamente cubiertas por los medios estatales de Vietnam. El número de declaraciones oficiales e informes de estos ilustran la importancia que el gobierno atribuyó a la disputa (Cotillon, 2017).

El sentimiento anti-China en Vietnam no solo se vinculó con la disputa marítima sino también con motivos económicos. La participación de China en proyectos en Vietnam en el sector recursos y energía ha sido polémico, y los medios nacionales han dado expresión a la desconfianza de la población hacia los intereses económicos de Beijing. Asimismo, otra área de preocupación en la población giró en torno al hecho de que las empresas chinas en Vietnam tienden a importar mano de obra china en lugar de contratar personal local (Morris Jung y Van Min, 2017). La combinación de estos factores dio lugar a una creciente retórica anti-China en la sociedad vietnamita, que se observó también en mensajes nacionalistas en las redes sociales (Cotillon, 2017).

Ante esta situación, el gobierno de Vietnam debió enfrentarse a un equilibrio entre lo nacional y lo internacional. A nivel doméstico, la población presionó al gobierno para que se mostrara firme ante el conflicto marítimo y buscara medios efectivos para salvaguardar la integridad territorial. Pero internacionalmente, Vietnam pretendió evitar que se agravara la situación mediante la adopción de un enfoque de conciliación con China, al considerar su rol como socio económico y su importancia geopolítica. Esta postura fue cuestionada e incluso calificada como una "subordinación" hacia Beijing (Strasakova, 2017, p. 8). Sin embargo, a partir del aumento de las tensiones en 2014, Hánoi hizo un giro significativo hacia una política de alianzas para contrarrestar la superioridad militar y creciente asertividad china. Para esto, trató de fortalecer las alianzas militares y de seguridad con Filipinas, Japón y Estados Unidos (Cotillon, 2017).

En términos oficiales, Vietnam y China mantuvieron una relación de compromiso mediante canales diplomáticos, militares y entre los respectivos partidos, con el objetivo de controlar las tensiones por la disputa territorial. Asimismo, 
los encuentros entre altos mandos de ambos países fueron frecuentes (Ton, 2018). Sin embargo, a nivel interno la situación fue distinta: tanto el gobierno de China como de Vietnam han celebrado conferencias de prensa nacionales para referirse a incidentes en el Mar del Sur de China, donde cada cual acusa a su contraparte. Pero ninguno de los dos realizó conferencias con el objetivo de anunciar avances positivos en las relaciones bilaterales (Kurlantzick, 2015).

Dado que China tiene un rol preponderante en la región y resulta un socio clave para sus intereses económicos, el gobierno vietnamita resolvió acomodarse a los fines de Beijing para sus propios beneficios, pero apuntando a no ser percibido como "pro-China" (Hung, 2018). Se puede considerar que la escalada del conflicto en el Mar del Sur de China, junto con el cambio en la opinión pública de la sociedad vietnamita, moldearon la percepción del gobierno de Vietnam respecto a China. Por lo tanto, un motivo que impulsó a Hanói a recurrir al balance interno de poder es la creciente tendencia negativa de la opinión pública ante las políticas expansionistas de China. Vietnam procuró mantener buenas relaciones con China, a medida que incrementaba su poder militar, pero viró hacia una estrategia de balance de poder más convencional a partir de 2014, con la intensificación del conflicto marítimo.

\section{El caso de Malasia: La ausencia de balance interno}

Malasia comparte las tensiones con Vietnam y demás países de la región por el conflicto en el Mar del Sur de China. Además, cabe destacar, durante la guerra fría China apoyó la insurgencia del Partido Comunista de Malasia que intentó derrocar al gobierno nacional (Sun, 2016). Siguiendo la lógica del realismo estructural, estos factores deberían haber impulsado a Kuala Lumpur a considerar el aumento de poder de China como una amenaza y a buscar restaurar el equilibrio de poder. Pero no fue así. De hecho, Malasia fue uno de los Estados que presentó menor gasto militar en la región asiática, pues mostró un descenso en los últimos años, a pesar de su creciente desarrollo económico. De 2013 a 2018, su gasto militar disminuyó casi un $20 \%$, incluso en términos de porcentaje de PBI: para 2013 destinaba un 1,5\% del PBI en gasto militar, mientras que para 2018 la cifra descendió a poco menos del 1 \% (SIPRI, 2019a).

La percepción de los gobiernos malayos parece ser el factor fundamental que explica este particular comportamiento. En el período previo, desde 1990 hasta 2012, las declaraciones de sus líderes reflejaron un bajo nivel de percepción de amenaza con respecto a Beijing, aun considerando su aumento de capacidades materiales (Kuik, 2013). Por otro lado, Malasia presenta, al igual que Vietnam, 
una relación cooperativa con los Estados Unidos. La cooperación bilateral se intensificó en términos de defensa, pero el gobierno malayo actuó de una manera extremadamente discreta, insistiendo en que no estaba totalmente alineado militarmente con el país (Kuik, 2015).

Con poco más de 30 millones de habitantes y una economía de aproximadamente el 0,4 \% del PBI mundial (Banco Mundial, 2019a), Malasia es un país relativamente pequeño. No obstante, desde su independencia en 1957, presentó un historial de desarrollo económico distintivo y exitoso que llevó a un cambio estructural $^{15}$ (Hill, 2012). Para Malasia, la seguridad se puede garantizar mediante el fomento del desarrollo de la región asiática en todos los aspectos. Si bien nunca ha sido un país que se haya inclinado hacia la fuerza militar, no significa que haya descuidado su desarrollo en defensa. Al contrario, su desarrollo militar siempre se movió junto a otros aspectos del crecimiento de la nación (Keling et al, 2011, p. 181). Su gasto militar en defensa se duplicó desde el 2000, cuando emprendió una serie de programas de adquisición de gran valor como la compra de tanques de batalla, lanzacohetes múltiples, aviones de combate y submarinos (Kaplan, 2014).

Asimismo, la transferencia de armas a Malasia aumentó, desde 2002-2006 hasta 2007-2011, casi un $300 \%$, por lo que el país se convirtió en uno de los mayores compradores de armas del Sudeste Asiático según el SIPRI. Esta modernización se vio impulsada por requisitos de seguridad, como la protección de la zona económica exclusiva en las aguas circundantes y el aumento de actividades en el Mar del Sur de China. Para Kaplan (2014), a diferencia de Vietnam, el proceso de modernización militar de Malasia no ha sido una expresión de nacionalismo. Esto se debe, en gran parte, al carácter multicultural de la sociedad malaya, lo cual provoca que no exista una sola identidad nacional. Estas tensiones étnicas al interior del país subvirtieron el desarrollo de un sentimiento nacionalista.

Al igual que en el caso de Vietnam, Malasia presenta cierta dependencia económica con China, por lo que también se puede calificar su relación como asimétrica. China es el mayor socio comercial de Malasia, mientras que Malasia es el mayor socio comercial de China en la ASEAN. Por lo tanto, la relación económica ha sido beneficiosa para ambas partes (Lean, 2016). Además, de acuerdo con datos provistos por la CIA, en 2017 el 20,6 \% de la población malaya es de etnia china, por lo que se puede relacionar la importancia que Malasia le otorga a China con cuestiones étnico-culturales (Central Intelligence Agency, 2020).

15 Con cambio estructural se hace referencia a la gran transformación económica del país, que supuso el cambio de una economía basada en recursos (con el caucho y estaño como los sectores dominantes para la exportación), a la producción manufacturera a gran escala. En comparación a 1970, la agricultura se redujo a un tercio, mientras que el sector industrial casi se duplicó (Hill, 2012). 
Por otro lado, un objetivo del gobierno de Najib consistió en atraer más inversiones chinas, además de fortalecer los lazos comerciales (Lean, 2016). Malasia es el país más receptivo de Asia respecto a la participación en OBOR: desde 2013, Malasia se convirtió gradualmente en un foco de la diplomacia de Beijing, debido a su ubicación estratégica entre los océanos Índico y Pacífico (Kuik, 2017). Asimismo, el acercamiento hacia China por parte de Malasia puede ser entendido como una consecuencia del aumento de la inversión china en el país y de las expectativas favorables respecto a la iniciativa OBOR (Rinehart, 2015).

En consideración de lo anterior, se puede interpretar la relativa dependencia económica de Malasia respecto a China como un factor explicativo de que Malasia no recurra a una estrategia de equilibrio de poder, aun manteniendo conflictos territoriales. No obstante, el caso de Vietnam ilustra justamente lo contrario: al igual que Malasia, es un país sensible económicamente al comportamiento chino, y cuyo crecimiento económico no se puede sostener sin la potencia. Pero esta dependencia económica no impide que Vietnam recurra a una estrategia de balance de poder. Por lo tanto, la relación entre la dependencia económica y el balance de poder no es lineal, sino que está mediada por el factor de la percepción de los respectivos gobiernos, que provoca que consideren -o no- a China como una amenaza para sus intereses.

Por otro lado, en 2015 la economía de Malasia experimentó una recesión significativa debido a una combinación de factores globales y nacionales, donde el más importante a nivel interno es el suceso del fondo de inversión estatal 1Malaysia Development Berhad (1MDB). Este consistió en un escándalo por la acusación del primer ministro Najib, en principio por mala administración de los fondos y, posteriormente, por corrupción (Parameswaran, 2016). Ante esto, un motivo del descenso del gasto militar pudo haber sido el esfuerzo de Malasia por reducir el déficit presupuestario y la deuda pública (SIPRI, 2019b). Pero la posterior recuperación económica no tuvo aparejada un nuevo incremento de este gasto, que incluso disminuyó en términos de porcentaje del PBI (Banco Mundial, 2019a; SIPRI, 2019a).

De esta manera, al igual que Vietnam antes de 2014, Malasia recurrió a una política exterior de hedging que involucró tanto a Estados Unidos como a China. La estrategia supuso no tomar partido entre las potencias y fue desarrollada, en primer lugar, con el objetivo de protegerse contra la potencial amenaza que podría resultar de China. En segundo término, se consideraron el peligro de conflicto y las preocupaciones domésticas asociadas a un gran poder como autoridad (Kuik, 2016; Rinehart, 2015). 


\section{Percepción del gobierno malayo: Etnia y legitimidad}

El aumento de poder de un Estado y la concepción por parte de otro Estado como una amenaza no resulta automático. Malasia no se enfocó, en términos de Brooks (1997), en la posibilidad de conflicto con China, sino que se basó en la probabilidad, permitiendo el desarrollo de otras cuestiones además de la seguridad. De este modo, la reacción de Malasia ante la probable amenaza de China en el Mar del Sur de China implicó el fomento de medidas pacíficas como la negociación, mediación y diplomacia (Alatas, 2016).

Asimismo, como se mencionó anteriormente, la asimetría de poder y la proximidad geográfica no tienen una lógica inherente propia: de acuerdo con el realismo neoclásico, los motivos que provocan que Estados más pequeños teman o se vean atraídos a un Estado en ascenso resultan de una combinación de factores domésticos. En el caso de Malasia, el factor étnico adquiere importancia considerable al momento de explicar las relaciones con China, dado que las minorías chinas son un segmento fuerte de la política nacional (Rinehart, 2015; Alatas, 2016).

Con la conformación de la coalición del Frente Nacional en 1973, se produjeron cambios políticos que limitaron el papel de las etnias no malayas en la vida política del país. Para equilibrar la situación, Najib llevó a cabo una política de acercamiento con China buscando estabilizar las relaciones entre los chinos y los malayos del país, además de controlar la insurgencia comunista en Malasia (Bakar, 2005). De esta manera, la legitimidad del Frente Nacional descansó, en cierta medida, en el gobierno chino. Por lo tanto, líderes sucesivos de la coalición (desde 1981 con Mahathir, Abdullah y hasta Najib en mayo de 2018) adoptaron una política pragmática hacia China y, por extensión, a las disputas marítimas. Esta política pragmática también fue impulsada por el deseo de maximizar los beneficios económicos y comerciales con China (Kuik, 2015).

En este punto se diferencia con Vietnam: mientras que en Malasia el 20,6 \% de la población es de etnia china, en Vietnam el porcentaje resulta inferior al 1 \% (Central Intelligence Agency, 2020). Asimismo, según datos del Asian Barometer Survey, en el período 2014-2016, el 75 \% de la población de Malasia consideró la influencia de China como positiva para su país. La cifra contrasta altamente con el caso de Vietnam, donde tan solo el $20 \%$ de su población la calificó como positiva (Chu y Chang, 2017).

Respecto a las relaciones económicas de Malasia con China, hubo visiones críticas por parte de la oposición y de grupos socioeconómicos del país que cuestionaron el marcado interés de Malasia de formar parte de OBOR. Kuik (2017), 
lo cual sugiere que el interés en participar en la iniciativa se puede haber debido a una estrategia desesperada de supervivencia del primer ministro Najib luego del escándalo de 1MDB. No obstante, la oposición planteó que la oleada de inversión china podría eventualmente socavar la soberanía nacional, dado que China adquirió activos estratégicos como la tierra y los puertos (Kuik, 2017).

Del mismo modo, si bien Malasia tiene intereses económicos en el Mar del Sur de China, sus reclamos no han sido tan frecuentes como otros Estados (Rinehart, 2015). En contraposición a Vietnam, Indonesia y Singapur, cuyos gobernantes plantearon la necesidad de garantizar el equilibrio de poder ante el crecimiento chino, los líderes de Malasia se refieren al auge de esta gran potencia como un "desafío" y no como una amenaza (Kuik, 2015). Además, en el conflicto marítimo, Malasia recurrió a una "diplomacia silenciosa": a pesar del aumento de las actividades chinas en aguas disputadas, el gobierno evitó informar al respecto a su población (Kreuzer, 2016). Asimismo, el gobierno malayo emitió protestas diplomáticas que se mantuvieron inicialmente confidenciales, aunque eventualmente las noticias aparecieron tanto en medios chinos como internacionales (Kreuzer, 2016).

En torno a esto, Kaplan (2014) sostiene que Malasia no concibe ningún país como una amenaza, lo que tiende a diluir el sentimiento nacionalista en el país. Esto puede ser un factor que explique por qué, a diferencia de Vietnam, Malasia no concibe a China como una amenaza. Sin embargo, hay que considerar que durante el período estudiado tuvieron lugar distintos acontecimientos en la política doméstica de Malasia, como la crisis económica de 2015 y el escándalo de corrupción de $1 \mathrm{MDB}$, que desviaron la atención sobre las amenazas externas.

Al considerar el conflicto como una probabilidad, el gobierno malayo permitió desarrollar otro tipo de relaciones con China, evitando entender la seguridad como el factor más urgente y teniendo en cuenta el factor étnico y la legitimidad que otorgaba el mero hecho de mantener buenas relaciones con Beijing. En ocasiones se intentó ocultar a su población los conflictos marítimos a partir de una diplomacia silenciosa. Mediante una política de hedging, el Frente Nacional enfatizó las oportunidades de converger con China, minimizando los desafíos de su creciente poder (Kuik, 2015). En el caso de Malasia, a pesar de las presiones externas, fueron los factores sociales domésticos y la propia dinámica de la política interna los que definieron los lineamientos de su política exterior. 


\section{Conclusiones}

Ante el aumento de poder de China, tanto en términos económicos como militares, el realismo estructural predeciría que los Estados de Asia tenderían al equilibrio de poder, para lo cual aumentarían su propio gasto militar. Sin embargo, esto no sucedió en todos los países de la región, lo cual plantea un interrogante sobre la teoría. Entre 2013 y 2018, por ejemplo, Vietnam y Malasia asumieron respuestas diferentes ante el aumento de poder de China; el primero aumentó su gasto militar un $38 \%$; el segundo disminuyo el suyo casi un $20 \%$, incluso en proporción a su PBI (SIPRI, 2019a). Este artículo avanzó sobre las razones de tal comportamiento mediante un estudio comparado de ambos casos, profundizó sobre el rol de las creencias y percepciones de los gobiernos como variable mediadora y planteó como hipótesis una fuerte incidencia de estos factores por sobre las presiones estructurales en el desarrollo de la política exterior.

Según los datos analizados, en el caso de Vietnam la hipótesis planteada fue corroborada, aunque con ciertos matices y no de la forma esperada: desde 2013 a 2018 se produjeron encuentros frecuentes entre los respectivos gobiernos, que generalmente mantuvieron una relación comprometida en distintos niveles de interacción y en canales diplomáticos y militares, como una manera de controlar las tensiones por la disputa en el Mar del Sur de China (Ton, 2018). Sin embargo, las buenas relaciones entre altos mandos del gobierno no se reflejan a nivel social: existe una creciente tendencia negativa de la opinión pública de Vietnam respecto a Beijing, principalmente a partir del incidente del 2014 en el Mar del Sur de China. A raíz de este hecho se intensificaron las protestas masivas contra China en distintas ciudades del país, que acrecentron, ampliamente, el nacionalismo y el sentimiento anti-China en la sociedad vietnamita (Tran, 2018).

El Partido Comunista de Vietnam debió enfrentarse a un dilema entre los intereses domésticos e internacionales: si bien a nivel interno la población instó al gobierno para que se mostrara asertivo y presionara en la disputa con Beijing, el gobierno vietnamita adoptó un enfoque de conciliación con China (Strasakova, 2017), procurando no ser percibido por su población como "pro-China" (Hung, 2018). En este sentido, la creciente tendencia negativa de la opinión pública respecto del comportamiento de China en el Mar del Sur de China es un factor que motivó al Partido Comunista de Vietnam hacia ejercer cierto grado de balance de poder. El histórico y creciente nacionalismo de la sociedad vietnamita y las relaciones entre el gobierno y la sociedad civil, ambas variables de carácter doméstico, también se constituyeron como un factor explicativo.

Por otro lado, en el caso de Malasia, se corroboró la hipótesis planteada: el gobierno malayo, influenciado sobre sus percepciones, distinguió entre la 
probabilidad y posibilidad de conflicto (Brooks, 1997), considerando a China como una amenaza poco probable, lo que le permitió centrarse en otras cuestiones. El Frente Nacional reflejó la voluntad política de priorizar la relación económica con China por sobre otros asuntos, evitando considerar la seguridad como el factor más urgente. De este modo, Malasia utilizó una política de hedging (al igual que Vietnam hasta 2014), que implicó no tomar partido entre China y Estados Unidos (Kuik, 2015).

Asimismo, el gobierno de Malasia debió considerar el factor étnico: las minorías étnicas chinas son un segmento fuerte de la política nacional, dado que históricamente China tiene un rol fundamental para el Frente Nacional de Malasia. La coalición obtenía legitimidad por el solo hecho de mantener relaciones cordiales con China. Frente a esto, en ocasiones el gobierno de Malasia intentó ocultar a la población los acontecimientos en el Mar del Sur de China mediante una estrategia de diplomacia silenciosa, que procuraba mantener las protestas diplomáticas en confidencialidad, para evitar una reacción hostil de su población (Kreuzer, 2016).

En los casos analizados, las distinciones en los factores étnicos y políticos de carácter doméstico resultaron fundamentales en el planteo y desarrollo de su política exterior. Abrir la "caja negra" del Estado mediante las herramientas teóricas del realismo neoclásico permite explicar sus diferentes comportamientos ante la presión sistémica de la estructura internacional.

En suma, el realismo neoclásico se presenta como un marco adecuado para explicar los casos analizados y resolver las incógnitas no resueltas por el realismo estructural en su vertiente original. Frente a esto, como futuras líneas de investigación, se sugiere dar continuidad al estudio de la interacción entre las presiones del sistema y las condiciones domésticas de los Estados en otros contextos y ámbitos temporales. Cabe preguntarse, en este sentido, qué sucederá en Malasia luego de la derrota del Frente Nacional y con la asunción de la coalición del Pacto de Esperanza respecto al poderío de China.

\section{Referencias}

Alatas, S. M. (2016). The South China Sea and Energy Security: Malaysia's Reaction to Emerging Geopolitical Reconfigurations. African and Asian Studies, 15(1), 33-52.

Amer, R. (2004). Assessing Sino-Vietnamese Relations through the Management of Contentious Issues. Contemporary Southeast Asia, 26(2), 320-345. 
Bakar, I. A. (2005). Malaysian perceptions of China. Historia Actual Online, 7, 93-105.

Banco Mundial (2019a). PBI (US\$ a precios constantes de 2010). https://datos.bancomundial.org/indicador/NY.GDP.MKTP.KD

Banco Mundial (2019b). Población, total. https://datos.bancomundial.org/indicador/sp.pop.totl

Battaleme, J. (2015). Cambiando el status quo de la geopolitica internacional: El acceso a los espacios comunes y las estrategias de negación de espacio y antiacceso. https://repositorio.uade.edu.ar/xmlui/handle/123456789/6367

BBC (2011). South China Sea: Vietnamese hold anti-Chinese protest. BBC News. https://www.bbc.com/news/world-asia-pacific-13661779

BBC (2012). Vietnam refuses to stamp new Chinese passports over map. BBC News. https://www.bbc.com/news/world-asia-20491426

BBC (2016). South China Sea: Vietnam airport screens hacked. BBC News. https:// www.bbc.com/news/world-asia-36927674

Beckley, M. (2017). The Emerging Military Balance in East Asia. How China's Neighbors Can Check Chinese Naval Expansion. International Security, 42(2), 78-119.

Brooks, S. G. (1997). Dueling Realisms. International Organization, 51(3), 445-477.

Buzan, B., y Cox, M. (2013). China and the US: Comparable cases of 'peaceful rise'? The Chinese Journal of International Politics, 6(2), 109-132.

Cai, P. (2017). Understanding China's belt and road initiative. Lowy Institute for International Policy, 1-22.

Central Intelligence Agency. (2020). The World Factbook. https://www.cia.gov/ library/publications/the-world-factbook/

Chen, S., y Feffer, J. (2009). China's military spending: soft rise or hard threat? Asian Perspective, 33(4) 47-67.

Chu, Y., y Chang, Y. (2017). Battle for Influence: Perceptions in Asia of China and the US. Global Asia, 12(1), 104-111.

Cotillon, H. (2017). Territorial Disputes and Nationalism: A Comparative Case Study of China and Vietnam. Journal of Current Southeast Asian Affairs, 36(1), 51-88.

Economy, E. (2018). China's new revolution: The reign of Xi Jinping. Foreign Affairs, 97, 60-74.

Gerring, J. (2001). Social Science Methodology: A Criterial Framework. Cambridge University Press. 
Hill, H. (2012). Malaysian economic development: looking backward and forward. Malaysia's development challenges: Graduating from the middle, 40.

Hung, N. M. (2018). VIETNAM IN 2017: Power Consolidation, Domestic Reforms, and Coping with New Geopolitical Challenges. Southeast Asian Affairs, 2018, 407-428.

Instituto Internacional de Estudios Estratégicos. (2018). The Military Balance. The Annual Assessment of Global Military Capabilities and Defence Economics.

Kaplan, R. D. (2014). Asia's Cauldron: The South China Sea and the End of a Stable Pacific. Ballantine Books.

Karim, M. F., y Chairil, T. (2016). Waiting for Hard Balancing?: Explaining Southeast Asia's Balancing Behaviour towards China. European Journal of East Asian Studies, 15(1), 34-61.

Keling, M.F., Ajis, M.N., Shuib, M.S., Othman, M.F., y Som, H.M. (2011). The Malaysian Government's Efforts in Managing Military and Defence Development. International Journal of Business and Social Science, 2(12), 180-192.

Kreuzer, P. (2016). A Comparison of Malaysian and Philippine Responses to China in the South China Sea. The Chinese Journal of International Politics, 9(3), 239-276.

$\mathrm{Ku}$, S. (2017). Strategies of China's Expansion and Taiwan's Survival in Southeast Asia: A Comparative Perspective. En L. Dittmer (Ed.), Taiwan and China, (pp. 249-279). University of California Press.

Kuik, C. (2013). Making Sense of Malaysia's China Policy: Asymmetry, Proximity, and Elite's Domestic Authority. The Chinese Journal of International Politics, 6(4), 429-467.

Kuik, C. (2015). Variations on a (Hedging) Theme: Comparing ASEAN Core States' Alignment Behavior. Joint U.S.-Korea Academic Studies, 26, 11-26.

Kuik, C. (2016). Malaysia Between the United States and China: What do Weaker States Hedge Against? Asian Politics \& Policy, 8(1), 155-177.

Kuik, C. (2017). Tempting Torch? Malaysia Embraces (and Leverages on) BRI Despite Domestic Discontent. Praxis: A Review of Policy Practice. Asian Politics \& Policy, 9(4), 652-654.

Kurlantzick, J. (2015). China-Vietnam Military Clash. Council on Foreign Relations.

Lean, H. H., y Smyth, R. (2016). The Malaysia-China economic relationship at 40: Broadening ties and meeting the challenges for future success. En H. Lean y R. Smyth (Eds.), Chinese global production networks in ASEAN (pp. 39-52). Springer International Publishing. 
Liff, A. P. y Ikenberry, G. J. (2014). Racing toward Tragedy? China's Rise, Military Competition in the Asia Pacific, and the Security Dilemma. International Security, 39(2), 52-91.

Lobell, S. E., Ripsman, N. M., y Taliaferro, J. W. (2009). Neoclassical Realism, the State, and Foreign Policy, Diplomacy \& Statecraft, 24(4), 691-693.

Mearsheimer, J. (2001). The Tragedy of Great Power Politics. W.W. Norton \& Company.

Ministerio de Relaciones Exteriores de Vietnam (19 de mayo de 2014). Peace, Stability in East Sea under Serious Threat, Says PM. www.mofa.gov.vn/en/ nr040807104143/nr040807105001/ns140512151537/view

Morris-Jung, J., y Min, P. (2017). Anti-Chinese Protest in Vietnam: Complex Conjunctures of Resource Governance, Geopolitics and State-Society Deadlock. En J. Morris-Jung (Ed.), China's Backyard: Policies and Politics of Chinese Resource Investments in Southeast Asia (pp. 229-255). ISEAS-Yusof Ishak Institute.

Morrison, W. M. (2014). China's economic rise: History, trends, challenges, and implications for the United States. Congressional Research Service.

Nguyen, T. (2016). Accommodating the dragon: Vietnam's enduring asymmetric entanglements with China. Hong Kong Baptist University.

Parameswaran, P. (2016). MALAYSIA IN 2015: Crises of Confidence. En M. Cook y D. Singh (Eds), Southeast Asian Affairs (pp. 183-197). ISEAS-Yusof Ishak.

Pham, D. N. (2016). From Marx to Market: The Debates on the Economic System in Vietnam's Revised Constitution. Asian Journal of Comparative Law, 11(2), 263-285.

Phi, V. (2012). Regional Development along Economic Corridors: Southern Coastal and Northern Sub-Corridors in Vietnam. En M. Ishida (Ed.), Emerging Economic Corridors in the Mekong Region. Bangkok Research Center.

Rinehart, I. E. (2015). Malaysia: Background and us relations. Congressional Research Service.

Ripsman, N. M., Taliaferro, J. W., y Lobell, S. E. (2016). Neoclassical realist theory of international politics. Oxford University Press.

Rocha Pino, M. (2020). La proyección de la Iniciativa Cinturón y Ruta de la Seda en la Unión Europea (2014-2019): Implicaciones económicas y normativas. Relaciones Internacionales, 93(2), 19-46. https://doi.org/10.15359/ri.93-2.1

Rose, G. (1998). Neoclassical Realism and Theories of Foreign Policy. World Politics, 51(1), 144-172. 
Rubbi, L. (2018). Guerra asimétrica: La estrategia de defensa de la República Popular China en el período 2012 - 2016 [Tesis de maestría]. Universidad Torcuato Di Tella.

Schweller, R. (2016). The Balance of Power in World Politics. Politics: Oxford Research Encyclopedias. 10.1093/acrefore/9780190228637.013.119

Schweller, R. (2018). Opposite but Compatible Nationalisms: A Neoclassical Realist Approach to the Future of US-China Relations. The Chinese Journal of International Politics, 11(1), 23-48.

Shoji, T. (2018). Vietnam's Security Cooperation with the United States: Historical Background, Present and Future Outlook. NIDS Journal of Defense and Security, 19, 3-16.

SIPRI. (2012). Trends in International Arms Transfers, 2011. SIPRI Fact Sheet.

SIPRI. (2018a). El gasto militar mundial se mantiene alto en 1,7 billones de dólares. https://www.sipri.org/sites/default/files/2018-05/milex_press_release_esp. pdf

SIPRI. (2018b). SIPRI Yearbook 2018: Armaments. Disarmament and International Security.

SIPRI. (2019a). Military Expenditure Database. https://www.sipri.org/databases/ milex

SIPRI (2019b). Trends in World Military Expenditure, 2018. SIPRI Fact Sheet.

South China Morning Post. (2014). Vietnam arrests demonstrators in renewed anti-Chinese protests. https://www.scmp.com/news/asia/article/1514821/ vietnam-arrests-demonstrators-renewed-anti-chinese-protests

South China Morning Post. (2014, 16 de mayo). In Vietnam's anti-Chinese protests, violence will only add fuel to fire. https:// www.scmp.com/comment/insight-opinion/article/1513049/ vietnams-anti-chinese-protests-violence-will-only-add-fuel

South China Morning Post. (2014, 18 de mayo). Vietnam arrests demonstrators in renewed anti-Chinese protests. https://www.scmp.com/news/asia/article/1514821/vietnam-arrests-demonstrators-renewed-anti-chinese-protests

Storey, I. (2018). Asia y el nuevo (des)orden mundial. En La era de la perplejidad. Repensar el mundo que conocíamos. BBVA. https://www.bbvaopenmind. com/wp-content/uploads/2018/01/BBVA-OpenMind-La-era-de-la-perplejidad-repensar-el-mundo-que-conociamos.pdf

Strasakova, M. (2017). Sino-Vietnamese Relations: Accommodating the Dragon? ISA International Conference 2017, University of Hong Kong. 
Sun, O. E., y Han, D. (2016). Malaysia's Relations with the Major Powers: China and the United States. S. Rajaratnam School of International Studies. http:// hdl.handle.net/11540/9268

Swaine, M. D. (2010). Perceptions of an Assertive China. China Leadership Monitor, 32(2), 1-19.

Thayer, C. A. (2018). Force Modernization: Vietnam. En D. Singh y M. Cook, Southeast Asian Affairs 2018 (pp. 429-444). ISEAS-Yusof Ishak.

Toai, D. B., Guan, X., y Ghimire, A. (2018). Situational Analysis of Vietnam for Belt and Road Initiative. Advances in Economics, Business and Management Research, 67,176-181.

Ton, A. D. (2018). Vietnam's Maritime Security Challenges and Regional Defence and Security Cooperation. Soundings, 4.

Tran, T. U. (2018). Coping with a rising power: Vietnam's hedging strategy toward China. Naval Postgraduate School.

Tuoi Tre News. (2016, 22 de enero). Vietnam will confront new maritime challenges, difficulties: military general. https://tuoitrenews.vn/politics/32895/vietnamwill-confront-new-maritime-challenges-difficulties-military-general

Vélez Serrano, M. (2018). De dragón revolucionario, a tímido, y ahora rejuvenecido. Relaciones Internacionales, 91(2), 1-23. https://doi.org/10.15359/ri.91-2.4

Walt, S. M. (1985). Alliance Formation and the Balance of World Power. International Security, 9(4), 3-43.

Walt, S. M. (1990). The origins of alliance. Cornell University Press.

Waltz, K. N. (1979). Theory of international politics. Addison-Wesley.

Womack, B. (2006). China and Vietnam: The Politics of Asymmetry. Cambridge University Press.

$\mathrm{Wu}, \mathrm{S}$. (2019). Vietnam: a case of military obsolescence in developing countries. The Pacific Review, 32(1), 113-130. 